\title{
Chronic constipation: building pathways for better bowel management
}

Professor Anton Emmanuel, Consultant Gastroenterologist at UCLH and the National Hospital for Neurology \& Neurosurgery

A recent review undertaken by the Bowel Interest Group (BIG) has shown that constipation is costing the NHS an alarming $f 71$ million per year in unplanned admissions. ${ }^{1}$ Yet, the condition is still underrecognised and under-supported in the National Health Service (NHS). Many of these unplanned admissions could be avoided if identified and managed at the primary care stage, but one of the key challenges is the absence of supporting information and materials for healthcare professionals treating the condition. Currently, the starting point for treatment is typically with standard Interventions, such as laxatives and dietary changes, but there is still little understanding of how to progress those patients' treatment beyond this. With 52,715 emergency admissions for constipation

in 2018 alone, ${ }^{2}$ this is a national problem that we are not talking about. This article is one small move towards redressing this issue, highlighting best practices in constipation management and providing a clear timeline of progression of treatments and insight into the associated costs of therapeutic alternatives.

\section{Breaking the taboo}

Chronic constipation affects nearly 2 million people in the UK - that is to say one in seven adults and one in three children. ${ }^{3}$ Yet its perceived taboo nature means it isn't always dealt with as effectively and promptly as it should. Our bowel habits are an important indicator of our health - so why aren't we giving the matter its due attention? The first challenge often lies in the initial step of speaking to a healthcare professional. In fact, nearly one in five people feel embarrassed talking to their GP about constipation, stating that they would try to solve it themselves without speaking to anyone about it. ${ }^{4}$ This communication void fuels a lack of awareness of what is 'normal' when it comes to bowel health and prevents early treatment, in turn increasing the likelihood of invasive interventions and unplanned hospital admissions. The corollary is that the high prevalence of the symptoms results in it being perceived by some healthcare professionals as low risk and of minor significance, thus exacerbating the communication gap.

\section{The risks of ignoring bowel health}

Constipation is a manageable and treatable condition, but without intervention, people with bowel disorders suffer from reduced quality of life including loss of independence and control, feelings of embarrassment, anxiety and depression as well as social isolation and impaired sexual relationships. Chronic constipation can also have unwanted side-effects such as urinary tract infections (UTIS) and failure to deal with it promptly can lead to complex problems such as haemorrhoids, anal fissures or rectal prolapse.

\footnotetext{
${ }^{1}$ Bowel Interest Group, Cost of Constipation Report, Second edition, 2019

2 Ibid

${ }^{3}$ Ibid

${ }^{4}$ Ibid
} 
Patients with nervous-system-related health conditions often suffer from neurogenic constipation in fact, approximately $68 \%$ of people with Multiple Sclerosis (MS) will develop bowel problems at some point. For these patients, constipation is an added cause of distress, making proper support especially important. Similarly, the third age is more likely to suffer from constipation, with approximately $26 \%$ of men and $34 \%$ of women aged 65 and above complaining of constipation. In order to regain control and optimize comfort, safety and privacy, it is critical to establish a bowel management program that is effective, timely, and sustainable with minimum physical and pharmacological interventions used.

\section{The Bowel Dysfunction Treatment Pyramid}

In response, many Trusts are developing dedicated bowel management pathways. In support, the Bowel Interest Group (BIG) has created a management pathway based on the NICE Clinical Knowledge Summary: the 'Bowel Dysfunction Treatment Pyramid'. The pyramid clearly indicates the length of time that each therapy should be tested before moving up to the next alternative. Effective bowel management requires a pathway that first tests each intervention and then, if they are ineffective over a strictly limited period, moves regularly up the pyramid until the patient's condition comes under control. Crucially, it is important to move as quickly as possible to the appropriate therapy level to fundamentally improve the patients' ability to live a normal and fulfilling life, both professionally and socially.

Conservative treatment options such as adjustment of diet/fluid intake, general lifestyle alteration, oral medications including stool softeners and laxatives, digital stimulation, suppositories and biofeedback constitute the base layer of the pyramid. If these are unsuccessful, the next step is to consider a minimally invasive treatment option such as transanal irrigation (TAI). The upper layers of the pyramid comprise the more invasive treatment options such as nerve stimulation implants and surgical colonic irrigation. Finally, if these are ineffective, a permanent stoma may be needed. Typically, each new treatment should be carried out for a period of three months before moving onto the next stage of intervention.

\section{The cost of constipation}

The Bowel Dysfunction Treatment Pyramid also provides useful indications of the cost to the NHS for each stage of treatment. From both a financial and patient-outcome perspective, there is no sense in lingering on an ineffective treatment echelon if it is not producing the desired results. In fact, the cost of standard starting treatment, which should amount to a minimum of $£ 2,539$, can reach up to $£ 32,298$ over seven years if practitioners do not move up the pyramid towards more effective treatment within the recommended timeframe. ${ }^{5}$ It is therefore crucial that practitioners take swift action to prevent the need for more serious action and additional costs in the long-term. Having this information will therefore be useful for GPs and Clinical Commissioning Groups in the responsible management of public funds.

There is a need for better bowel care in the UK. Unfortunately, constipation continues to be seen as a low priority condition despite affecting large numbers of the population and enormously diminishing the quality of life of those it affects. Adopting effective bowel management at the primary level

\footnotetext{
${ }^{5}$ Ibid
} 
reduces emergency admissions and bed occupancy, thereby alleviating some of the pressure on the NHS as well as improving the quality of life for patients. This will require a national change of attitude about the diagnosis and management of constipation throughout the NHS.

Please find the Bowel Interest Group's full report - Dealing with Chronic Constipation: Information for General Practitioners - here 